\title{
Higgs field and cosmological parameters in the fractal quantum system
}

\author{
Valeriy Abramov* \\ Donetsk Institute for Physics and Engineering named after A.A. Galkin, Ukraine
}

\begin{abstract}
For the fractal model of the Universe the relations of cosmological parameters and the Higgs field are established. Estimates of the critical density, the expansion and speed-up parameters of the Universe (the Hubble constant and the cosmological redshift); temperature and anisotropy of the cosmic microwave background radiation were performed.
\end{abstract}

The hypothesis of the hierarchical structure of the Universe (HHSU) became the basis for fractal cosmology [1]. The development of the technique of spatial scanning made it possible to determine the speed of expansion of the Universe, the cosmic distances to the far Galaxies with great accuracy [2]. As separate elements of large-scale fractal structures of the Universe (SELFSU), Galaxies, clusters and superclusters of Galaxies, the largest supercluster - Great Attractor, walls, filaments, voids are considered [1]. HHSU makes it possible to use models of active nanoelements (fractal dislocations, quantum dots with variable parameters [3]) to describe SELFSU [4]. The aim of the work is to establish the connections between cosmological parameters and the Higgs field within the framework of the fractal model of the Universe. For the unit cell (such as the effective atom and the Higgs boson) model relationships, that relate the Hubble constant, the cosmological redshift, the temperature and the anisotropy of the relic radiation are obtained. The shift of the cosmic microwave background $(\mathrm{CMB})$ frequency is described by equations

$$
\delta \nu_{\gamma}=4 Q_{H 2} \gamma u_{\Omega 1}^{2} / N_{0}^{\prime} ; \delta \nu_{\gamma}^{\prime}=N_{0}^{\prime} \delta \nu_{\gamma} / N_{02}^{\prime} ; u_{\Omega 1}^{2}=\left(\gamma \dashv \xi_{q} \mid\right) / 2 \gamma ; \xi_{q}=\nu_{r 1}-\nu_{r 2} ; \Omega_{c 1}^{\prime}=4|\lambda|^{2} N /\left.\xi_{q}\right|^{2} .
$$

Using from [4] the values $Q_{H 2}=1 / 3, \nu_{r 1}=160.3988698 \mathrm{GHz}$ (the maximum of the CMB frequency), $N_{0}^{\prime}, N_{02}^{\prime}, \nu_{r 2}=142.8161605 \mathrm{GHz}$ (the shifted radiation frequency), we find the frequency shifts $\delta \nu_{\gamma}=3.3920592 \mathrm{kHz}, \delta \nu_{\gamma}^{\prime}=3.4281246 \mathrm{kHz}$ and the density of cold dark matter $\Omega_{c 1}^{\prime}=0.228071512$. The value $\delta \nu_{\gamma}^{\prime}$ (received with the Higgs field) agrees with the experiment on shifting the NMR frequency (3.42 kHz) for superfluid helium-3 [5]. Nonlinear equations (determine the parameters of the CMB) for functions $Q_{G}, B_{G}$ are

$$
Q_{G}=Q_{H}\left(1-Q_{H}\right)^{2} /\left(1+Q_{H}\left(1-Q_{H}\right)^{2}\right) ; \quad B_{G}=1-2 Q_{G} ; \quad V_{Q}=d Q_{G} / d Q_{H} ; \quad A_{Q}=d V_{Q} / d Q_{H}
$$

Corresponding author: vsabramov@mail.ru 
The dependences of these functions on $Q_{H}$ are given in Fig. 1. Variant $V_{Q}>0$ and $A_{Q}>0$ corresponds to the accelerated expansion of the Universe [4]. In model I [4] $Q_{H 0}=H_{01} / H_{02}$, where $H_{01}, H_{02}$ are the Hubble constants.
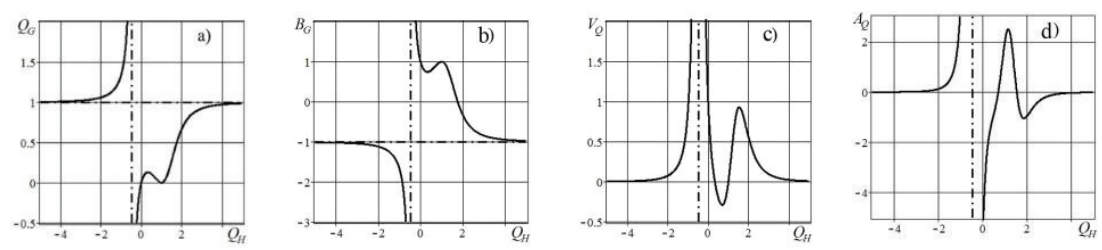

Fig. 1. The behavior of functions $Q_{G}$ (a), $B_{G}$ (b), $V_{Q}$ (c), $A_{Q}$ (d) on $Q_{H}$.

The modeling results of the deformation field $\left(u_{2}\right)$ for a coupled system (dislocationquantum dot) are given on fig. 2. The function $u_{2}(n, m)$ is complex with a pronounced stochastic behavior and essentially depends on the relationships between the characteristic dimensions $N_{1} \times N_{2}$ of the investigated region, the values of the semi-axes $n_{c 2}, m_{c 2}$ at the quantum dot (fig. 2). Fractal holes with proportional semi-axes (fig. 2 a), elongated along the axis $O n$ (fig. 2 b) and along the axis $\mathrm{Om}$ (fig. 2 c) in elliptical structures are observed inside the region of wave behavior (fig. 2 a,b,c). In this case, a pronounced phenomenon of the anisotropy of the fractal hole appears. The character of the anisotropy for effective damping (fig. 2 d,e,f) agrees with the anisotropy of the fractal hole.

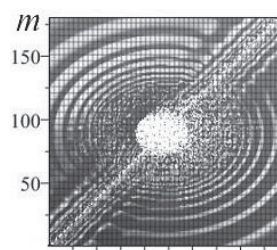

$50 \quad 100 \quad 150 \quad 200 \quad n$

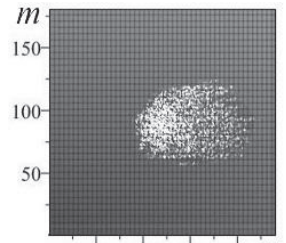

$50 \quad 100 \quad 150 \quad 200 n$ a)

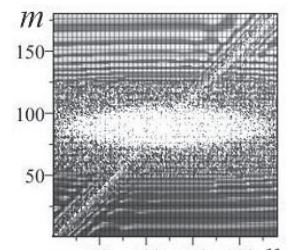

$50 \quad 100 \quad 150 \quad 200 n$

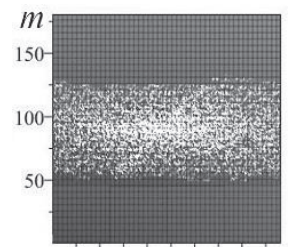

$50 \quad 100 \quad 150 \quad 200 n \quad \mathrm{e}$

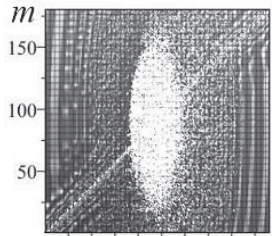

$\left.\begin{array}{llllll}50 & 100 & 150 & 200 & n & \mathrm{C}\end{array}\right)$

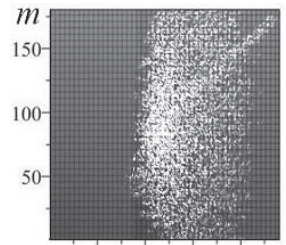

$\begin{array}{llllll}50 & 100 & 150 & 200 & n & \mathrm{f})\end{array}$

Fig. 2. Cross-sections $\operatorname{Re}\left(u_{2}\right) \in[-10 ; 10]$ (a,b,c), $\operatorname{Im}\left(u_{2}\right) \in[-10 ; 10]$ (d,e,f) (top view) for coupled system with variable modules $k_{\mathrm{i}}$ for $u_{2}$ and semi-axes for quantum dot: (a, d) $3 n_{c 2} ; 3 m_{c 2}$; (b, e) $12 n_{c 2} ; 3 m_{c 2} ;(\mathrm{c}, \mathrm{f}) 3 n_{c 2} ; 12 m_{c 2}$.

\section{References}

[1] B. Novosyadlyy, Universe, Space, Time 6(143), 4 (2016)

[2] A.G. Riess, A.V. Filippenko et al., Astronomical Journal 116, 1009 (1998)

[3] V.S. Abramov, Bull. RAS: Physics 80(7), 859 (2016)

[4] V.S. Abramov, Bull. of Donetsk National University A(1), 36 (2017)

[5] Superfluidity in Helium-3: Collection of Articles (Moscow, Mir, 1977) 